\title{
Computational Evaluation of Potential Correction Methods for Unicoronal \\ Craniosynostosis
}

Selim Bozkurt, $\mathrm{PhD}^{1,2}$, Alessandro Borghi, $\mathrm{PhD}^{2,3}$, Owase Jeelani, FRCS (NeuroSurg) $^{2,3}$, David Dunaway, FRCS (Plast) ${ }^{2,3}$, Silvia Schievano, $\mathrm{PhD}^{1,2}$

1. University College London, Institute of Cardiovascular Science, Department of Children's Cardiovascular Disease

2. University College London, Institute of Child Health, Department of Developmental Biology and Cancer

3. Great Ormond Street Hospital for Children

Address correspondence and reprint requests to Dr Selim Bozkurt, PhD, UCL Great Ormond Street Institute of Child Health, 30 Guilford Street, London WC1N 1EH, UK; E-mail: s.bozkurt@ucl.ac.uk

Tel: +44 (0) 2076792000

Fax: +44 (0)20 72429789

This research was supported by Great Ormond Street Hospital Children's Charity through the FaceValue programme grant (no. 508857), the Engineering and Physical Sciences Research Council award (EP/N02124X/1) and the European Research Council Starting Grant (CAD4FACE/757923). This work was undertaken at GOSH/ICH, UCLH/UCL who received a proportion of funding from the United Kingdom Department of Health's NIHR Biomedical Research Centre funding scheme. The funders had no role in study design, collection, analysis and interpretation of the data, decision to publish, or preparation of the manuscript. 


\section{Abstract}

2 Unicoronal craniosynostosis is the second most common type of non-syndromic 3 craniosynostosis: it is characterised by ipsilateral forehead and fronto-parietal region

4 flattening with contralateral compensatory bossing. It is a complex condition, therefore 5 which is difficult to treat because of the asymmetry in the orbits, cranium and face. The 6 aim of this study is to understand optimal osteotomy locations, dimensions, and force

7 requirements for surgical operations of unicoronal craniosynostosis using a patient8 specific finite element model and - at the same time - to evaluate the potential 9 application of a new device made from Nitinol which was developed to expand the affected side of a unicoronal craniosynostosis skull without performing osteotomies.

11 The model geometry was reconstructed using Simpleware ScanIP. The bone and sutures were modelled using elastic properties to perform the finite element analyses in MSc Marc software. The simulation results showed that expanding the cranium without osteotomy requires a significant amount of force. Therefore, expansion of the cranium achieved by Nitinol devices may not be sufficient to correct the deformity. Moreover, the size and locations of the osteotomies are crucial for an optimal outcome from surgical operations in unicoronal craniosynostosis.

Keywords: unicoronal craniosynostosis, finite element modelling, patient-specific model 


\section{Introduction}

Unicoronal craniosynostosis is the second most common type of nonsyndromic craniosynostosis ${ }^{1}$, characterised by ipsilateral forehead and fronto-parietal region flattening with contralateral compensatory bossing. It is a complex condition, aesthetically difficult to treat because of the asymmetry in the orbits, cranium and face ${ }^{2}$ and can cause neurological complications due to the irregular cranial geometry and relatively high intracranial pressure ${ }^{3}$.

Unicoronal craniosynostosis is corrected surgically between six and twelve months of age, with the aim of expanding and reshaping the skull ${ }^{4}$. The primary surgical method is fronto-orbital advancement by reshaping of the bones at the front of the skull and above the eye sockets to normalise the head shape $e^{5,6}$. This approach may not offer optimal aesthetical outcomes for middle and lower facial asymmetry and ocular anomalies may be persistent ${ }^{6,7}$. Endoscopic-assisted craniosynostosis surgery is offered as an alternative to open surgeries to minimise these complications ${ }^{8}$ and it is followed by four to six months of helmet therapy ${ }^{9}$. This endoscopic technique assisted by helmet therapy improves the facial growth and symmetry significantly ${ }^{10}$. However, it generally yields suboptimal results in patients older than six months ${ }^{8}$. Distraction osteogenesis following suturectomy is another approach for unicoronal craniosynostosis treatment, allowing displacement of the bones in the skull apart from each other into a controlled, optimal position ${ }^{11,12}$. Positive outcomes have been reported with fewer complications when compared to fronto-orbital advancement ${ }^{13}$. Nonetheless, a comparison of the outcome of this technique with controls remains to be studied ${ }^{5,14}$. 
1 Understanding the causes of suboptimal correction or predicting the correction

2 in a skull may help to improve the outcome of the correction techniques used in

3 unicoronal craniosynostosis skulls. Computational finite element modelling have

4 already been used to simulate craniosynostosis in patient skulls and can provide a

5 valuable tool to design osteotomies and understand forces affecting the bones ${ }^{15}$

6 Moreover, results from the numerical simulations can be used to develop new devices

7 to correct the craniosynostosis or optimise the existing devices to improve the outcome

8 of the treatments ${ }^{16}$.

The aim of this study is to use a patient-specific and parametric finite element model to study osteotomy locations, dimensions, and force requirements on a unicoronal craniosynostosis skull, in order to understand distraction forces and displacement outcomes.

\section{Materials and Methods}

An eight-month old boy with unicoronal craniosynostosis was selected for this study. Simpleware ScanIP was used to reconstruct the skull bone volume and sutures from the patient computed tomography images (Figure 1, top panels), to create a finite element model of the patient skull (tetrahedral elements with -25 coarseness option, generating around 440000 structural 3D elements) and to perform osteotomies on the reconstructed model geometries. The skull materials were modelled as linear elastic with Young's modulus $(E)$ and Poisson's ratio $(v)$ of the bony part set to $157 \mathrm{MPa}$ and 0.22 respectively, and Young's modulus $(E)$ and Poisson's ratio $(v)$ of the sutures set to $8.3 \mathrm{MPa}$ and 0.49 respectively, according to the child's age ${ }^{17,18}$. Fixed boundary conditions describing no displacements and rotations were applied at the base of the 
1 skull model similar to ${ }^{16}$. Computational simulations were carried out in MSc Marc

2 Mentat using an implicit solver.

As an initial trial, the reconstructed skull geometry was used to evaluate the deformations imposed by a $0.5 \mathrm{MPa}$ surface load applied as boundary condition to a portion of the inner surface of the affected side of the skull, without osteotomies (Figure 1 , bottom panels). The surface load represents the force exerted by a potential shape memory alloy device as presented $\mathrm{in}^{19}$, fixed to the outer portion of the skull on the affected side. The device would recover its initial shape whilst puling the bone outwards, thus correcting the skull geometrical abnormality.

Correction of the unicoronal craniosynostosis skull by means of osteotomies was studied using three different set of cuts varying in size and location. Distraction was obtained by applying nodal displacements to the finite element model cuts, aiming to correct the deformity as in fronto-orbital advancement. The nodal displacements represent the effect of potential devices such as the craniofacial springs described in ${ }^{20}$ Results were evaluated in terms of displacements and stresses.

The first type of osteotomies (T1) was performed on the affected side of the skull in conjunction with a cut through the orbits (Fig. 2a). The cut on the side and top of the skull was changed by $10 \mathrm{~mm}$ steps towards the frontal bone. The cut in the orbits was aligned to the nasion and also moved by $10 \mathrm{~mm}$ steps. The length of the osteotomies was changed to evaluate the effect of the osteotomy sizes on the displacement of the cut bones, along with osteotomy locations.

The second type of osteotomy (T2) aimed to correct the affected side on the patient's skull by a cut on the lateral side, aligned to the coronal suture, and frontal bone above the orbit, and by dividing the bone portion into three smaller parts for 
1 independent distraction (Fig. 2b). The cut bones were also trimmed at the edge to

2 avoid non-anatomical shape when distracted (Fig. 2c).

The third set of osteotomies (T3) included a cut aligned with the coronal suture on the affected side and continuing to the vicinity of the frontozygomatic suture (Fig. 2d). This cut was extended around the orbit to evaluate the effect of this osteotomy variations (Fig. 2e).

The nodal displacements applied in the simulations are listed in Table S1 and the directions are shown in Figure 3, for the presented bone displacements in the result section. The skull displacements and stresses are presented for five different cases which summarise the general response of the skull under various cut sizes and locations for each osteotomy analysed using computational simulations.

\section{Results}

The displacement map for the skull model corrected by a surface load is shown in Figure 4.

Loading the frontal bone with $0.5 \mathrm{MPa}$ surface pressure overall expanded the cranial vault. The deformation on the affected side of the frontal bone provided a more natural look whilst a slight collapse was recorded on top of the affected side of the skull. The orbits were deformed because of the displacements occurring in the skull.

The bone displacement maps for the first type of the evaluated osteotomies are shown in Figure 5.

Relatively shorter cuts on the side and top of the skull, and in the orbits caused a slight collapse of the top of the skull (Fig. 5I-III). Extending the cuts in the orbits reduced the displacement of the top of the skull (Fig. 5IV). The most extended cut at the top of the skull (Fig.5V) caused almost no deformation of the top of the skull and 
1 corrected the frontal bone better with respect to relatively shorter cuts. There was no

2 displacement on the unaffected side of the skull for model $\mathrm{V}$ because of the extended

3 cuts in the orbits, and on the side and top of the skull.

4 The displacement maps for the second type of selected osteotomies are shown in $5 \quad$ Figure 6.

7

Distracting the separated bones only in one direction on the horizontal axis caused misalignment between the separated bones and the rest of the skull (Fig. 6I). Imposing displacement in two directions and moving the bones on a horizontal plane improved the alignment between the cut bones and the rest of the skull (Fig. 6II). Nonetheless, the alignment between the cut bones and the skull depends on optimal adjustment of the predefined displacements, as further increase of these displacements may result in misalignment between the cut bones and the rest of the skull (Fig. 6III). However, higher displacement values or imposing predefined displacements in three axes can be used to nicely correct the frontal bone on the affected side of the unicoronal craniosynostosis. Indeed, the trimming of the cut bones at the edges allowed avoiding misalignment and imposing different displacements as boundary conditions. Applying displacements on the three axes for the cut bones provides better correction for the affected side of the frontal bone (Fig. 6V). The displacement maps for the third type of osteotomies are given in Figure 7.

In the third type of evaluated osteotomies imposing relatively higher displacement in the cut bone towards the front of the skull increases the displacement in the cut part; however, this also causes a misalignment between the cut bone and the rest of the skull. Extending the cut made on the side and top of the skull causes a 
1 collapse on the non-affected part of the cut bone and creates abnormal skull shape 2 (Fig. 7V).

The corresponding maximum principal stresses on the skull model for the evaluated osteotomies are shown in Figure 8.

The maximum stress for the first type of osteotomies (T1) did not change significantly with the extended cuts in the orbits when cut on the side was furthest back on the skull. The stress increased when the cut on the side of the skull was moved towards the frontal bone. Aligning the cut with the suture reduced the stress whilst extension of the cut on the top skull increased the stress.

In the second set of osteotomy simulations (T2), the maximum stress showed a correlation with the alignment of the cut bones and the skull. The stresses on the skull for the trimmed bones was lower for the imposed displacements with respect to the stresses on the skull for the non-trimmed bones. Moreover, trimming the bones caused a better alignment between cut bones and the skull.

The maximum stress for the third set of osteotomies (T3) was relatively higher for the extended cut at the vicinity of the frontozygomatic suture. The stress distribution remained relatively low for the cuts until the frontozygomatic suture. Extending the cut on top of the skull reduced the stress further for this type of osteotomy. The stress levels remained significantly higher for third type of the evaluated osteotomy (T3) with respect the first and second type of osteotomies (T1, T2).

\section{Discussion}

In this study, outcomes of potential different correction techniques for unicoronal craniosynostosis were evaluated using a patient-specific computational model. The first simulated treatment technique was the application of an innovative 
1 Nitinol device to correct the affected side of the skull without performing osteotomies ${ }^{19}$.

2 Nitinol is a super-elastic shape memory alloy used widely in clinical applications ${ }^{21}$, as

3 it can recover its initial shape after being largely deformed ${ }^{22}$. This property would be

4 ideal to achieve controlled displacement and therefore optimal shape outcome on the

5 affected side of the unicoronal craniosynostosis skull Correction of the skull deformity

6 with such device would depend on the amount of the device recovery, which in turns

7 would depend on the mechanical response of the bone (i.e. bone properties such as elastic modulus) to the force exerted by the device. The effect of the simulated device, which aimed at pulling outwards the affected side of the frontal bone, expanded the cranial vault and provided a more natural look for the skull. However, the overall load required for deformity correction in this patient specific case was found too high to be achieved with the simulated device. Using larger sizes or thicker plates may not be suitable because of the limited skull space. In younger children, with softer skull bones, the forces applied by this potential device may expand the cranium in the affected side, however, the correction may not be satisfactory. In older children, osteotomies are deemed necessary for correction of unicoronal craniosynostosis.

Three different types of osteotomies were evaluated in this study. The first included cuts on the side and top of the skull and in the orbits. Longer cuts yielded a better correction and a more natural look for the cut part without causing deformities on rest of the skull. However, performing such osteotomies requires open and highly invasive surgical techniques because of the cut in the orbits. Therefore, implantation of devices developed for minimally invasive approaches such as springs as in the sagittal craniosynostosis or Nitinol meshes could not work with this type of osteotomies. 

side of the frontal bone in the skull by dividing it into three smaller parts to distract them separately. Careful arrangement of the displacement forces is required for bone distraction to obtain an accurate correction in the frontal portion on the affected side, as distracting the bones only in one direction may cause misalignments between the cut bones and the skull (Fig. 6). Patient specific surgical planning, potentially using the described methodology, would allow careful selection of this osteotomy lines and device placement for optimal shape outcome.

The third type of evaluated osteotomies could be delivered using minimal invasive surgical techniques. Also in this case, the length of the cut on the side and top of the skull played a significant role in bone displacement. Extending the cut on the side and top of the skull yielded an abnormal skull shape because of the minimal cut on the side of the skull (Fig. 7V). Extending the cut through the orbits as shown in Fig. 5V might yield better shape result. However, as mentioned before, this would require open surgical techniques instead of minimal invasive approaches. Moreover, the stress on the skull is relatively high for this type of osteotomy.

In this study, various osteotomies and possible spring placement locations were investigated using computational modelling in a patient specific model. The simulation results are valid for the specific patient skull model. The proposed methodology may help define optimal osteotomy size and locations in any unicoronal patient skull before the surgical operation, becoming standard of care in the future. It should be noted that 3D printed skull models have already been used for surgical planning ${ }^{23}$. However, the mechanical properties of 3D printing materials are significantly different with respect to mechanical properties of infant skull making 3D printed in-vitro models unsuitable to evaluate clinical scenarios similar to the presented computational models ${ }^{17,24,25}$. 
1 Nonetheless, computational modelling is a powerful tool and allows to assess and compare outcome of new techniques resorting to in-vivo assessment.

As a main limitation it should be noted that this is an initial study and clinical applications would require further analyses to consider spatial and biological responses. Factors such as bone thickness, location of fusion, differences in biologic response to stretch or remodelling of bone, and the influence of the abnormal cranial base deformity on the overlying skull structure would all play a role in the final outcome. Nonetheless the potential of the presented methodology, finite element analysis, for surgical planning is shown in this initial work to address clinical effectiveness in normal tissues, at least in the earliest stages of treatment. In the future, the preliminary model presented in this study will be further elaborated to include more case, anatomy and structure variability, and the biological processes such as calcification of the osteotomies or change in the material properties over time in order to achieve improved outcome prediction.

\section{Conclusions}

Patient specific computational models of skulls with unicoronal craniosynostosis can provide information to support the design of new devices and potentially guide treatment planning. The simulation results showed that expanding the cranium without osteotomy requires a significant amount of force, difficult to deliver using minimally invasive devices.

The effects of the displacements applied after performing different osteotomies and the immediate outcome of different surgical operations can be quantified using finite element models. The simulation results gave valuable insights about the size of the cuts made and displacements occurred on the studied skull. 
1. Garza RM, Khosla RK. Nonsyndromic craniosynostosis. Semin Plast Surg. 2012; 26:53-63. <https://doi.org/10.1055/s-0032-1320063>.

2. Rodriguez ED, Losee JE, Neligan PC. Plastic Surgery: Volume 3: Craniofacial, Head and Neck Surgery and Pediatric Plastic Surgery. Elsevier, 2017.

3. Spruijt B, Tasker RC, Driessen C, Lequin MH, van Veelen MLC, Mathijssen IMJ, Joosten KFM. Abnormal transcranial Doppler cerebral blood flow velocity and blood pressure profiles in children with syndromic craniosynostosis and papilledema. Journal of Cranio-Maxillofacial Surgery. 2016; 44:465-470. <https://doi.org/10.1016/j.jcms.2016.01.001>.

4. Persing JA. MOC-PS(SM) CME article: management considerations in the treatment of craniosynostosis. Plast Reconstr Surg. 2008; 121:1-11. $<$ https://doi.org/10.1097/01.prs.0000305929.40363.bf>.

5. Alford J, Derderian CA, Smartt JM. Surgical Treatment of Nonsyndromic Unicoronal Craniosynostosis. J Craniofac Surg. 2018; 29:1199-1207. <https://doi.org/10.1097/SCS.0000000000004509>.

6. Balaji SM. Unicoronal Craniosynostosis and Plagiocephaly Correction with Fronto-orbital Bone Remodeling and Advancement. Ann Maxillofac Surg. 2017; 7:108-111. <https://doi.org/10.4103/ams.ams_80_17>.

7. Tan SPK, Proctor MR, Mulliken JB, Rogers GF. Early frontofacial symmetry after correction of unilateral coronal synostosis: frontoorbital advancement vs endoscopic strip craniectomy and helmet therapy. J Craniofac Surg. 2013; 24:1190-1194. <https://doi.org/10.1097/SCS.0b013e318299742e>.

8. Honeycutt JH. Endoscopic-Assisted Craniosynostosis Surgery. Semin Plast Surg. 2014; 28:144-149. <https://doi.org/10.1055/s-0034-1384810>.

9. Berry-Candelario J, Ridgway EB, Grondin RT, Rogers GF, Proctor MR. Endoscope-assisted strip craniectomy and postoperative helmet therapy for treatment of craniosynostosis. Neurosurg Focus. 2011; 31:E5. <https://doi.org/10.3171/2011.6.FOCUS1198>.

10. Cartwright CC, Jimenez DF, Barone CM, Baker L. Endoscopic strip craniectomy: a minimally invasive treatment for early correction of craniosynostosis. J Neurosci Nurs. 2003; 35:130-138.

11. Park DH, Yoon SH. Craniofacial malformation treatment: craniosynostosis and positional plagiocephaly. Journal of the Korean Medical Association. 2012; 55:878-886. <https://doi.org/10.5124/jkma.2012.55.9.878>.

12. Shen W, Cui J, Chen J, Buffoli B, Rodella LF, Zou J, Ji Y, Chen H. Piezosurgical Suturectomy and Sutural Distraction Osteogenesis for the Treatment of Unilateral Coronal Synostosis. Plast Reconstr Surg Glob Open. 2015; 3. <https://doi.org/10.1097/GOX.0000000000000382>. 
13. Tahiri Y, Swanson JW, Taylor JA. Distraction Osteogenesis Versus Conventional Fronto-Orbital Advancement for the Treatment of Unilateral Coronal Synostosis: A Comparison of Perioperative Morbidity and Short-Term Outcomes. J Craniofac Surg. 2015; 26:1904-1908. <https://doi.org/10.1097/SCS.0000000000002020>.

14. Brandel MG, Dalle Ore CL, Reid CM, Zhu W, Lance S, Meltzer H, Gosman AA. Distraction Osteogenesis for Unicoronal Craniosynostosis: Rotational Flap Technique and Case Series. Plast Reconstr Surg. 2018; 142:904e-908e. <https://doi.org/10.1097/PRS.0000000000005053>.

15. Malde O, Libby J, Moazen M. An Overview of Modelling Craniosynostosis Using the Finite Element Method. MSY. 2019; 10:74-82. $<$ https://doi.org/10.1159/000490833>.

16. Borghi A, Rodriguez-Florez N, Rodgers W, James G, Hayward R, Dunaway D, Jeelani O, Schievano S. Spring assisted cranioplasty: A patient specific computational model. Med Eng Phys. 2018; 53:58-65. $<$ https://doi.org/10.1016/j.medengphy.2018.01.001>.

17. Li Z, Liu W, Zhang J, Hu J. Prediction of skull fracture risk for children 0-9 months old through validated parametric finite element model and cadaver test reconstruction. Int J Legal Med. 2015; 129:1055-1066. <https://doi.org/10.1007/s00414-015-1190-6>.

18. Li Z, Zhang J, Hu J. Surface material effects on fall-induced paediatric head injuries: a combined approach of testing, modelling and optimisation. International Journal of Crashworthiness. 2013; 18:371-384. <https://doi.org/10.1080/13588265.2013.801291>.

19. Borghi A, Rodgers W, Schievano S, Ponniah A, Jeelani O, Dunaway D. Proof of Concept Study for the Design, Manufacturing, and Testing of a Patient-Specific Shape Memory Device for Treatment of Unicoronal Craniosynostosis. $J$ Craniofac Surg. 2018; 29:45-48. <https://doi.org/10.1097/SCS.0000000000004025>.

20. Borghi A, Schievano S, Rodriguez Florez N, McNicholas R, Rodgers W, Ponniah A, James G, Hayward R, Dunaway D, Jeelani NUO. Assessment of spring cranioplasty biomechanics in sagittal craniosynostosis patients. $J$ Neurosurg Pediatr. 2017; 20:400-409. <https://doi.org/10.3171/2017.1.PEDS16475>.

21. Duerig T, Pelton A, Stöckel D. An overview of nitinol medical applications. Materials Science and Engineering: A. 1999; 273-275:149-160. <https://doi.org/10.1016/S0921-5093(99)00294-4>.

22. Wadood A. Brief Overview on Nitinol as Biomaterial. Advances in Materials Science and Engineering. https://www.hindawi.com/journals/amse/2016/4173138/. Accessed January 27, 2019. 
23. LoPresti M, Daniels B, Buchanan EP, Monson L, Lam S. Virtual surgical planning and 3D printing in repeat calvarial vault reconstruction for craniosynostosis: technical note. Journal of Neurosurgery: Pediatrics. 2017; 19:490-494. <https://doi.org/10.3171/2016.10.PEDS16301>.

24. Decuir F, Phelan K, Hollins BC. Mechanical Strength of 3-D Printed Filaments. In: 2016 32nd Southern Biomedical Engineering Conference (SBEC). 2016, pp 47-48.

25. Subramaniam SR, Samykano M, Selvamani SK, Ngui WK, Kadirgama K, Sudhakar K, Idris MS. Preliminary investigations of polylactic acid (PLA) properties. AIP Conference Proceedings. 2019; 2059:020038. <https://doi.org/10.1063/1.5085981>. 


\section{Figure Legends}

Figure 1. The reconstructed patient-specific and finite element models with unicoronal craniosynostosis, the shaded area with light blue represents the Nitinol device pulling the bones outwards direction to expand the cranium.

Figure 2. The performed T1 (a), T2 (b, c) and T3 (d, e) type osteotomies on the skull model

Figure 3. The direction of the predefined displacements for the evaluated osteotomy types (T1, T2 and T3) and in the presented finite element models (I-V)

Figure 4. The displacements in the unicoronal craniosynostosis skull model corrected by a surface load

Figure 5. The displacements on the skull model for the first type of the evaluated osteotomy (T1), I-V show the skull models as given in Figure 2

Figure 6. The displacements on the skull model for the second type of the evaluated osteotomy (T2), I-V show the skull models as given in Figure 2

Figure 7. The displacements on the skull model for the third type of the evaluated osteotomy (T3), I-V show the skull models as given in Figure 2

Figure 8. The maximum principal stresses for the evaluated osteotomies, the arrows point notches on the osteotomies where stresses are concentrated 
Figure 1

Click here to access/download;Figure (1 Figure per File);Figure_1.tif $\underline{\underline{ \pm}}$
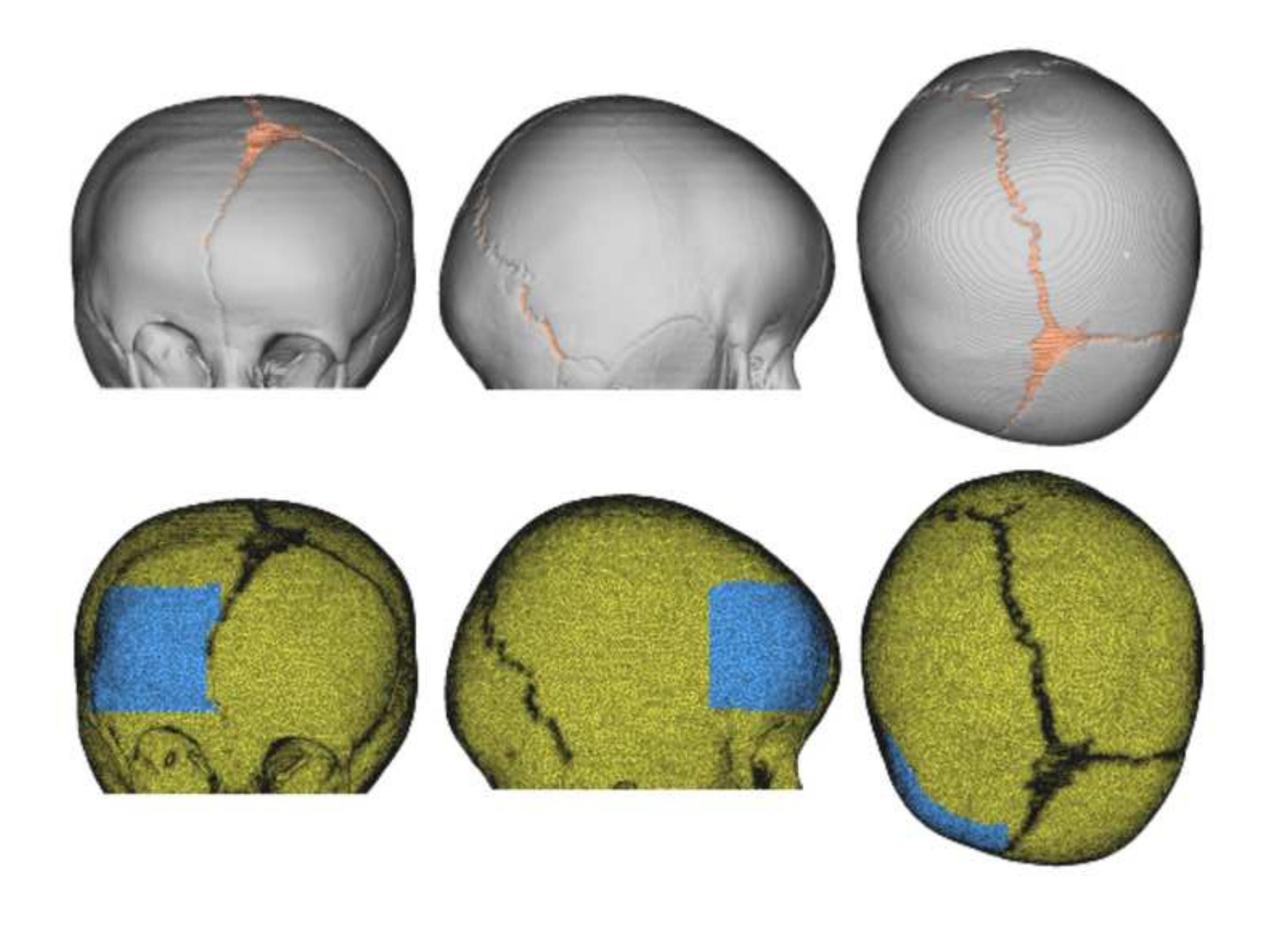


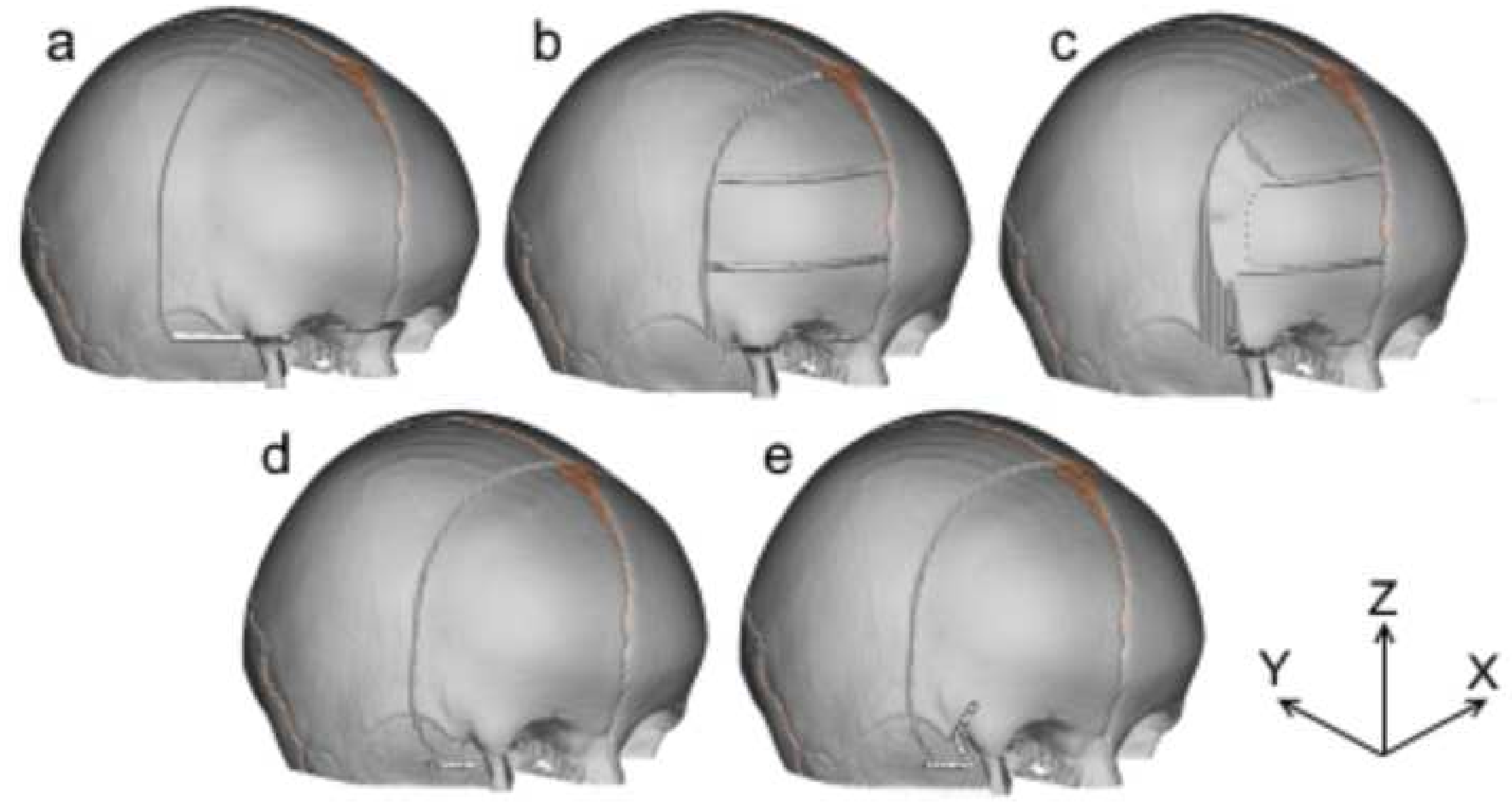



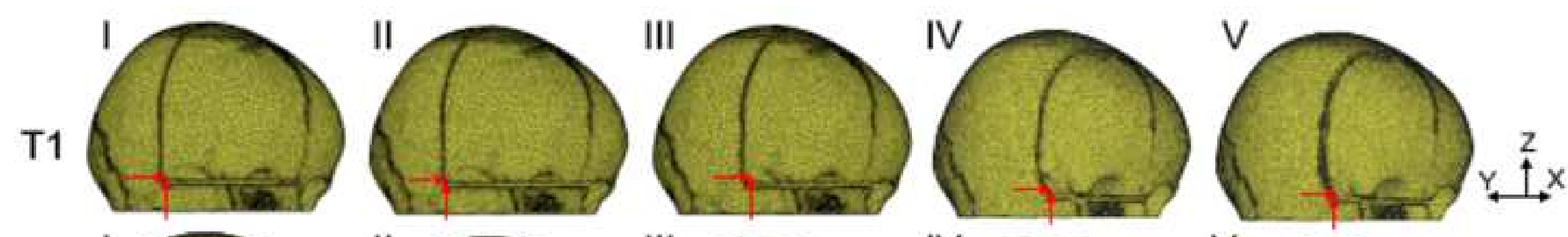

T2
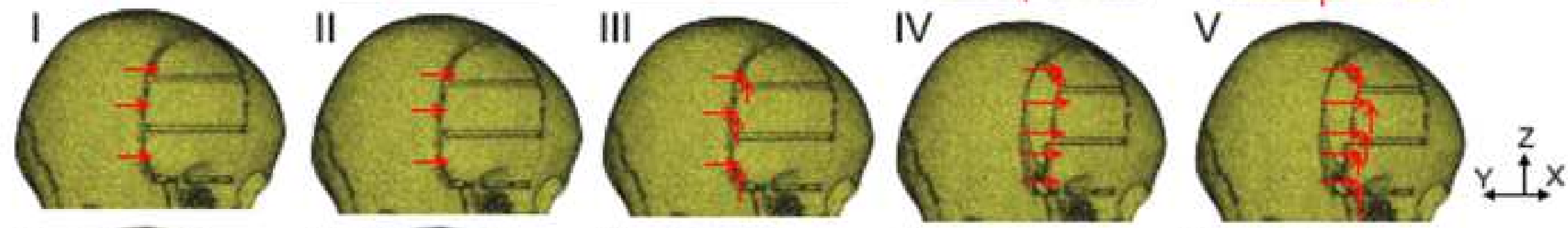

T3
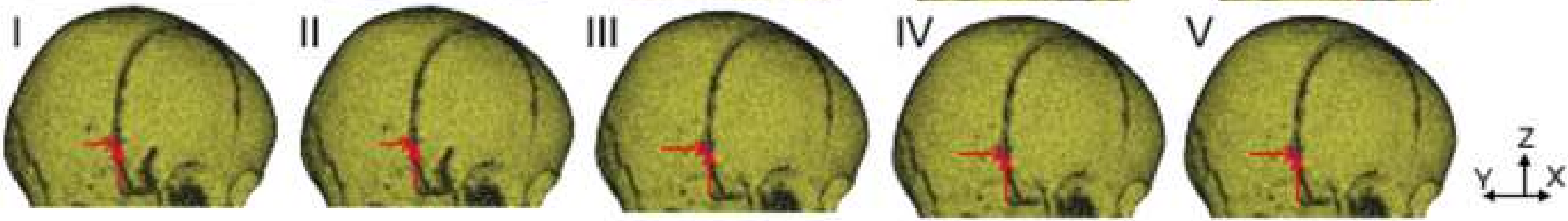

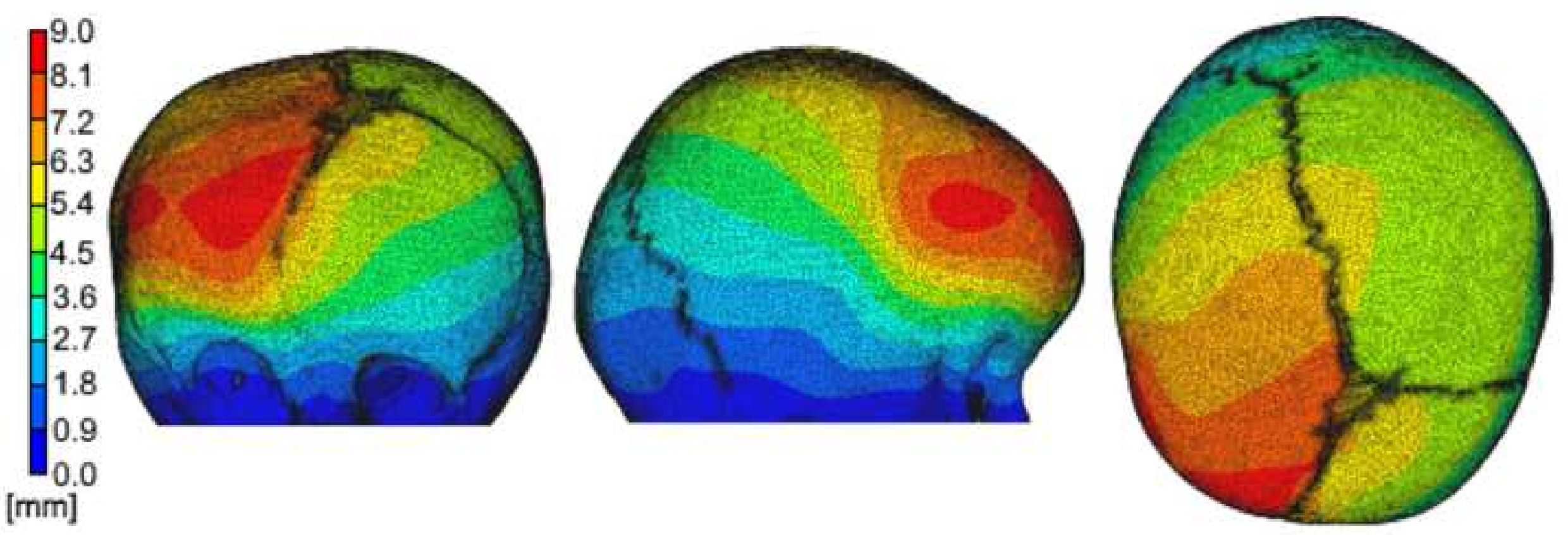


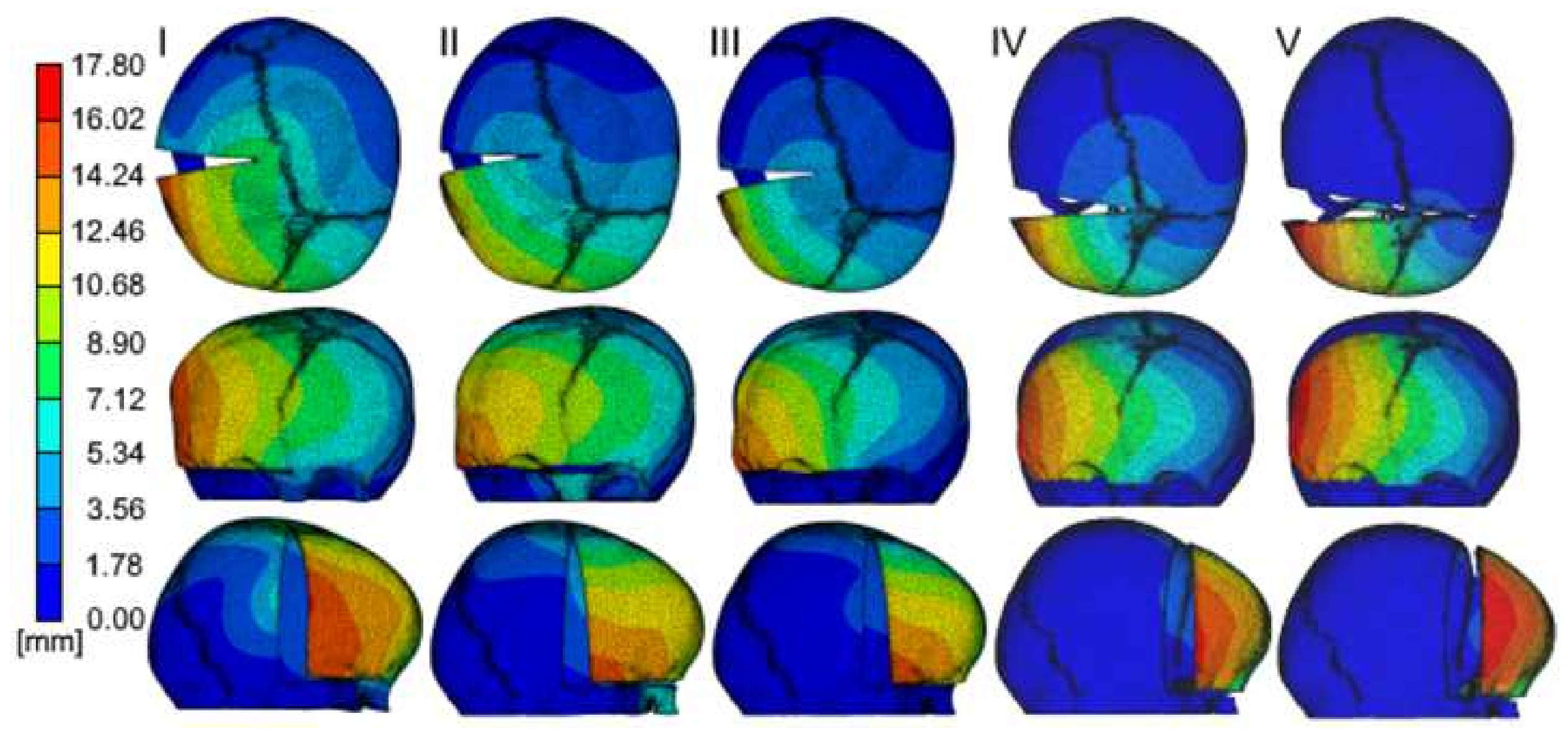




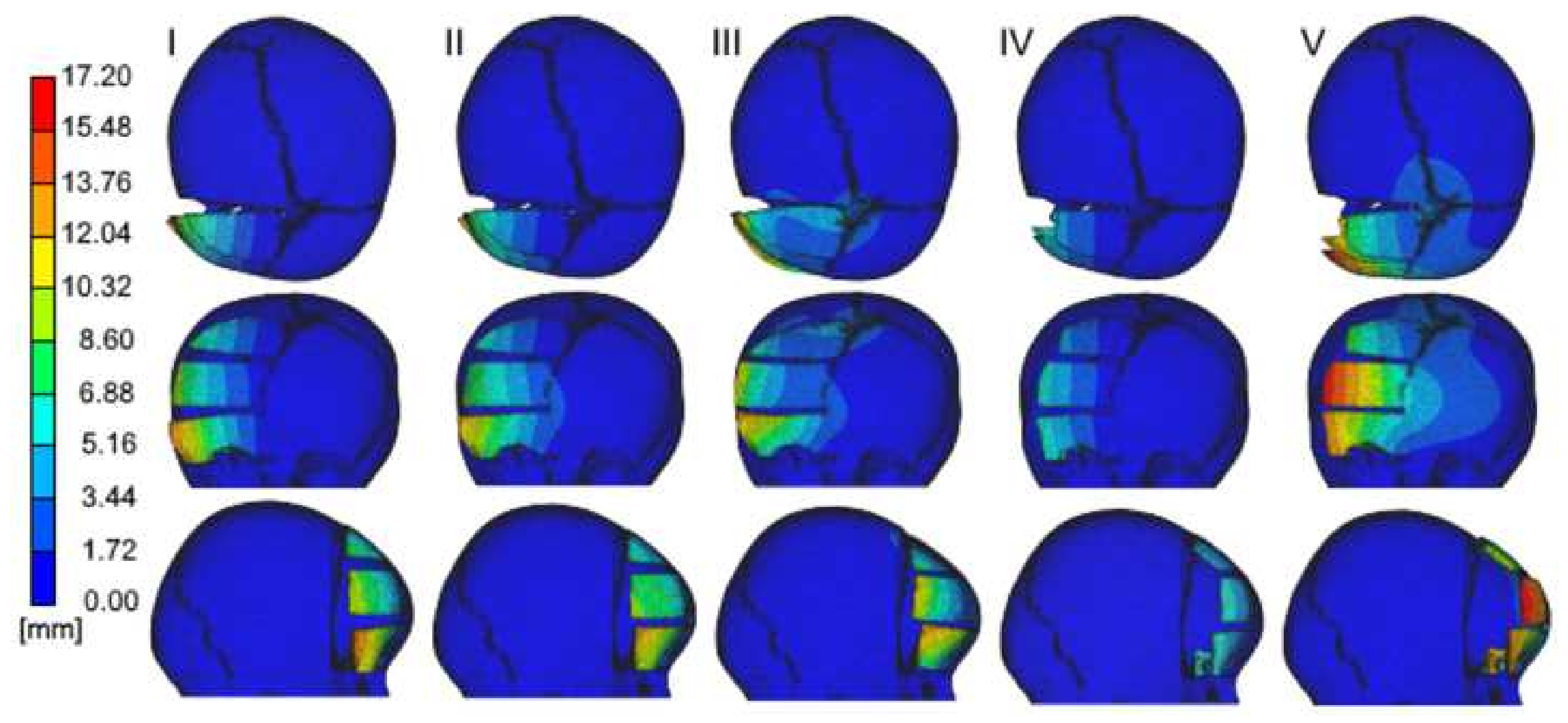




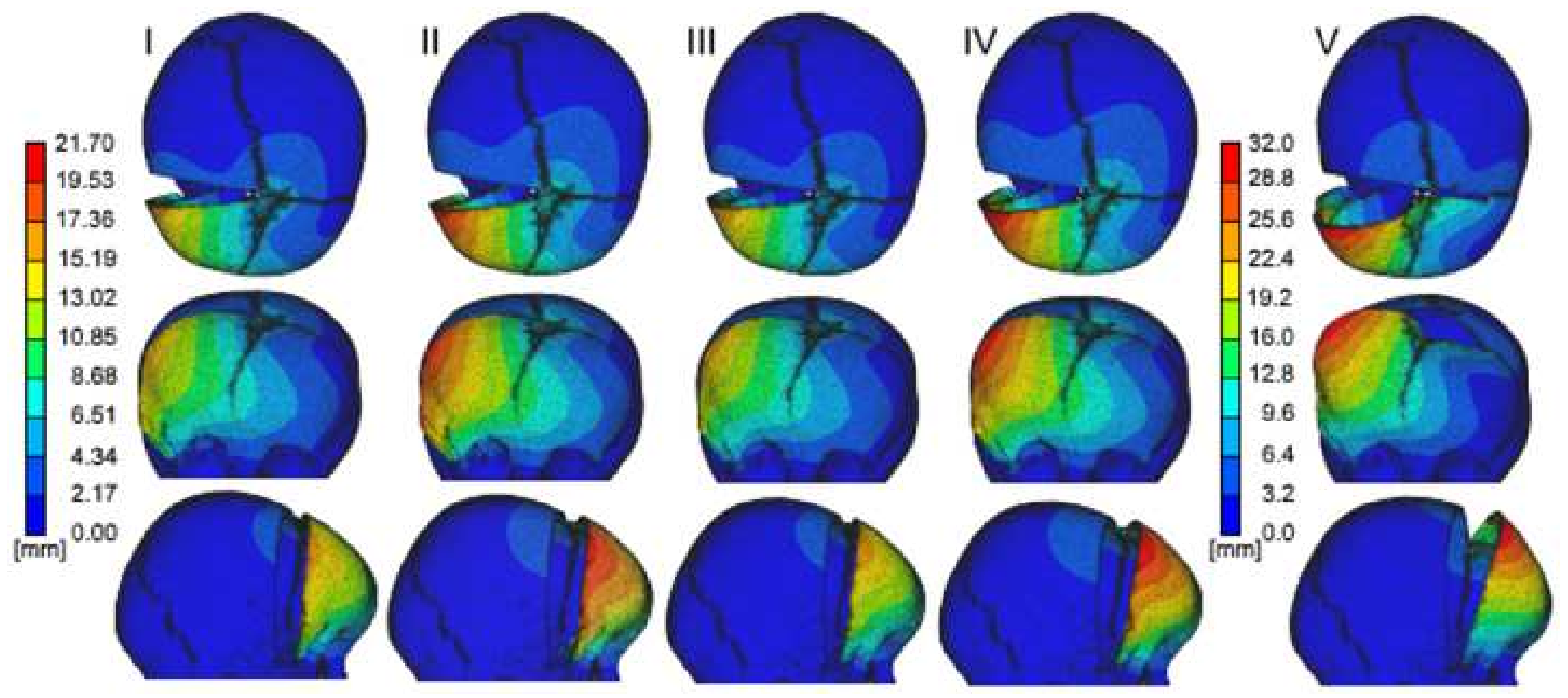




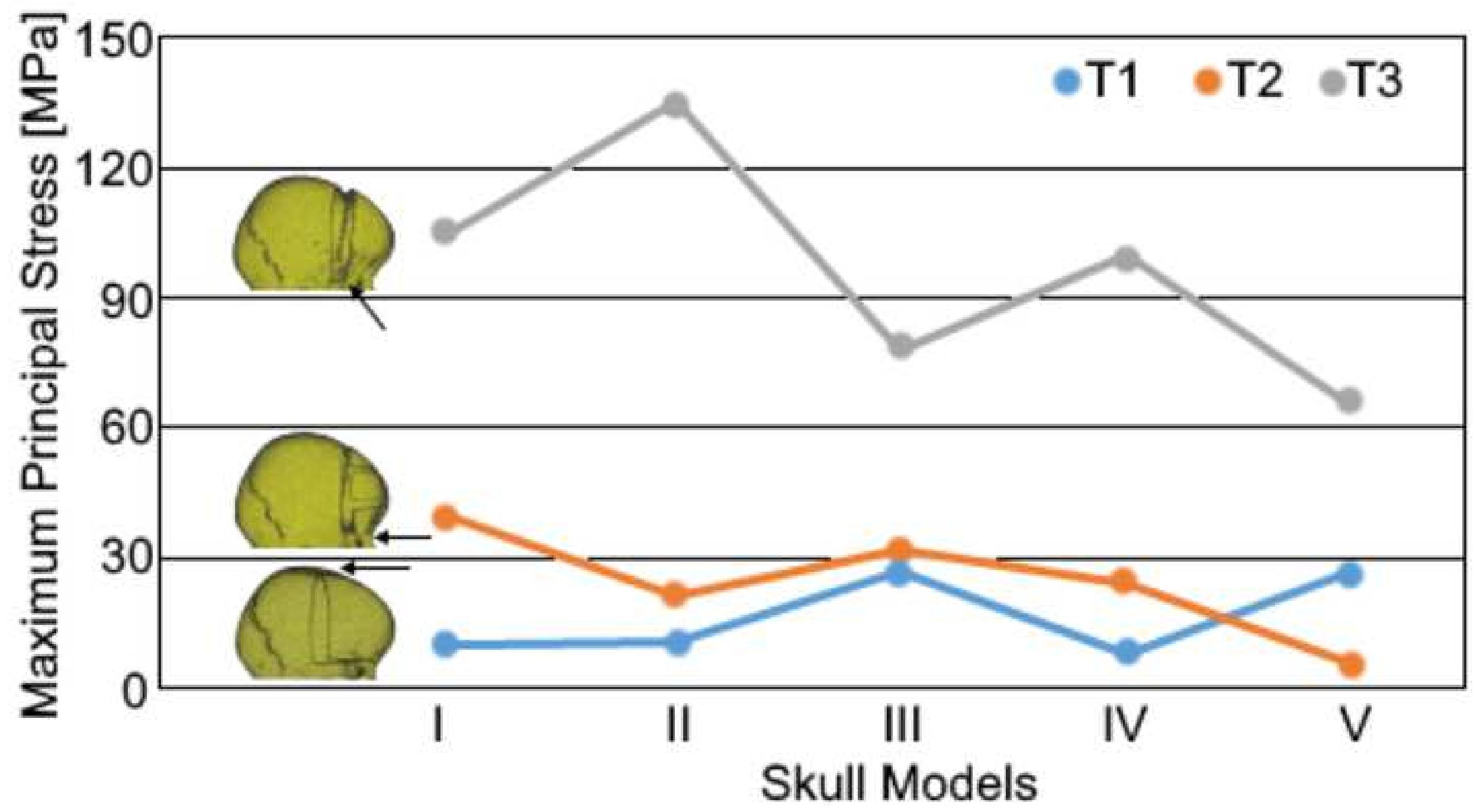

